\title{
Clinical efficacy analysis of Osimertinib treatment for a patient with leptomeningeal metastasis of EGFR+ non-small cell lung cancer without the T790M mutation
}

\author{
Xueyang $\mathrm{Hu}^{1}$, Wenjun Chen ${ }^{2}$, Xiaoqiu $\mathrm{Li}^{1}$, Chenchen Zhao ${ }^{1}$, Congjun Zhang ${ }^{1}$, Fuxing Xiong ${ }^{1}$, Hongyang Wu ${ }^{1}$ \\ ${ }^{1}$ Department of Medical Oncology, The First Affiliated Hospital of Anhui Medical University, Hefei 230031, China; ${ }^{2}$ Phase I Clinical Center, Anhui \\ Chest Hospital, Hefei 230022, China \\ Contributions: (I) Conception and design: H Wu, X Hu; (II) Administrative support: H Wu; (III) Provision of study materials or patients: X Li, \\ C Zhao, C Zhang, F Xiong; (IV) Collection and assembly of data: W Chen; (V) Data analysis and interpretation: H Wu, X Hu , W Chen; (VI) \\ Manuscript writing: All authors; (VII) Final approval of manuscript: All authors. \\ Correspondence to: Hongyang Wu, MD, PhD. Department of Medical Oncology, The First Affiliated Hospital of Anhui Medical University, Hefei \\ 230031, China. Email: wuhoya1970@163.com.
}

Background: To find the method of therapy of leptomeningeal metastasis (LM) to non-small cell lung cancer (NSCLC) patient with EGFR mutation (EGFR+) but without T790M mutation.

Methods: A retrospective analysis was reviewed for 5 NSCLC patients with EGFR+ who develop to LM from January 2018 to February 2019 in our hospital.

Results: All five NSCLC cases were adenocarcinoma, four cases were verified existed EGFR mutation with 19 exon deletion in the first diagnosed by biopsy tissue, the other tissue was verified 21 exon mutation. Two cases were initially diagnosed with LM, and the other three cases were found metastasis with leptomeningeal respectively after 64, 3 and 4 months when the lung cancer was diagnosed. There were not verified to exist T790M mutation with EGFR+ when all the five cases developed to LM. The major symptom was headache and blurred vision. In the image scanning, two cases were not revealed, but other three cases show that multiple metastatic lesions with brain and meninges. All patients were identified existed adenocarcinoma cells in cerebrospinal fluid (CSF). Four cases were treated by the first epidermal growth factor receptor tyrosine kinase inhibitor (EGFR-TKI) and joint therapy including chemotherapy and radiotherapy, and the other case was treated by temozolomide and intrathecal chemotherapy in their earlier therapy. The curative effect was significant when they took osimertinib orally $80 \mathrm{mg}$ once a day, for the disease progressing. The neurological symptoms were relieved in patient about 5-10 days after osimertinib treatment. The remission time was 10, $7,7,5,4$ months respectively until last following time to June 2019. The survival time was respectively 74, 7, 27, 18, and 4 months. The side effects were not increased.

Conclusions: Whether EGFR+ with T790M mutation was positive or negative, osimertinib is an effective drug and can improve quality of life and prolong the survival for NSCLC patient with EGFR mutation to progress LM.

Keywords: Osimertinib; T790M negative; non-small cell lung cancer (NSCLC); leptomeningeal metastasis (LM); clinical efficacy

Accepted for publication Sep 10, 2019. Accepted for publication Oct 17, 2019.

doi: 10.21037/apm.2019.10.13

View this article at: http://dx.doi.org/10.21037/apm.2019.10.13 


\section{Introduction}

Lung cancer metastases to the brain are a common phenomenon. Most of them present as brain parenchymal lesions, but, simple leptomeningeal metastasis (LM) or cerebrospinal fluid (CSF) metastasis is rare (1). Brain metastasis often showed the patient had poor prognosis, but it can be improved by cranial radiotherapy and intracranial chemotherapy. However, simple LM or CSF metastasis often lack effective imaging evidence (2), some hospitals do not have the methods to test CSF, that lead the diagnosis exceedingly difficult for these patients, and lacked effective treatment method, so the prognosis became worse. In recent years, with the widespread application of comprehensive treatment for lung cancer, the survival rate of patients has gradually improved, and the probability of LM has also increased (3). More than $10 \%$ of patients with non-small cell lung cancer (NSCLC) with epidermal growth factor receptor mutations $(E G F R+)$ have been reported to show LM (4). As a third-generation epidermal growth factor receptor tyrosine kinase inhibitor (EGFR-TKI), osimertinib had been shown effective in patients with T790M mutationpositive LM (5). However, there are few reports on the treatment of osimertinib in T790M negative patients with LM of lung cancer (6). We reported the clinical data of 5 patients with EGFR mutated NSCLC with LM treated in our department from January 2018 to February 2019, and analyzed the treatment process and outcome of osimertinib, to seek effective methods for the treatment of such patients.

\section{Methods}

This retrospective analysis was approved by The Clinical Medical Research Ethics Committee of The First Affiliated Hospital of Anhui Medical University. This study was only concluded about clinical cases, image data and laboratory results, without experimental animals and human tissues. The privacy consent was achieved by all patients. A retrospective analysis was reviewed for 5 NSCLC patients with EGFR+ whose develop to LM from January 2018 to February 2019 in the Department of Medical Oncology, the First Affiliated Hospital of Anhui Medical University. Total survival was obtained by outpatient or telephone follow-up to June 2019. Through the analysis of previous treatment regimens, disease changes, imaging manifestations related to LM, CSF testing, gene testing and blood CEA changes of 5 patients, the efficacy of osimertinib orally $80 \mathrm{mg}$ once a day in treating EGFR+ NSCLC with LM and its relationship to $T 790 M$ were studied.

\section{Results}

\section{Clinical and imaging findings}

Clinical data were shown in Table 1.

Among the 5 patients with LM of lung cancer, 2 were male, and 3 were female. The median age was 43 years ( 38 to 62 years). LM was found in 2 patients at the first diagnosis, and in the other 3 patients, LM occurred about 64,3 , and 4 months after the diagnosis of lung cancer. All the 5 patients presented with non-localized neurological symptoms, including cranial hypertension headache and dizziness, and 3 patients presented with cranial nerve involvement, blurred vision, and decreased vision. These imaging findings of 5 patients were different (Figure 1), among which 2 patients were negative with no signs of brain and LM (Figure 1C,E), 3 patients suggested multiple enhanced foci of brain parenchyma and leptomeninges (Figure $1 A, B, D$ ), and Figure $1 F$.

\section{CSF examination}

Adenocarcinoma cells were detected in CSF in all the 5 patients, and biochemical examination of CSF suggested that glucose was decreased, the protein was increased, and CEA was increased compared with normal value (Table 2).

\section{Gene testing}

High-throughput sequencing (NGS) of puncture tissues and peripheral blood was performed in 5 patients after the diagnosis of LM, which improved the gene detection. All cases showed negative T790M.

\section{Treatment regimen and survival}

LM was present in 2 of the 5 patients at first diagnosis; the gene detection results of the two patients were 19 exon deletion and T790M negative. One patient was treated with intrathecal chemotherapy (methotrexate + cytarabine) combined with temozolomide, but the symptoms of headache and dizziness were not significantly relieved. When the treatment was replaced by osimertinib orally $80 \mathrm{mg}$ once a day and the neurological symptoms disappeared significantly 10 days later. The other patient developed intracranial lesions after gefitinib combined with 
Table 1 Five cases clinical data

\begin{tabular}{|c|c|c|c|c|c|}
\hline Sex & Male & Female & Female & Female & Male \\
\hline $\begin{array}{l}\text { Source of tumor } \\
\text { tissue }\end{array}$ & $\begin{array}{l}\text { Lumbar vertebra } \\
\text { puncture }\end{array}$ & Lumbar puncture & Lung puncture & $\begin{array}{l}\text { Right cervical } \\
\text { lymph node } \\
\text { puncture }\end{array}$ & Lung puncture \\
\hline EGFR status & 19 exon deletion & 19 exon deletion & 19 exon deletion & 19 exon deletion & 21 exon mutation \\
\hline $\begin{array}{l}\text { Treatment before } \\
\text { osimertinib }\end{array}$ & $\begin{array}{l}\text { Pemetrexed + } \\
\text { cisplatin; erlotinib }\end{array}$ & $\begin{array}{l}\text { Intrathecal chemotherapy } \\
\text { (methotrexate + cytarabine); } \\
\text { temozolomide }\end{array}$ & $\begin{array}{l}\text { Gefitinib; temozolomide; } \\
\text { whole brain radiotherapy }\end{array}$ & $\begin{array}{l}\text { Pemetrexed + } \\
\text { cisplatin; gefitinib }\end{array}$ & $\begin{array}{l}\text { Pemetrexed + } \\
\text { cisplatin; icotinib }\end{array}$ \\
\hline $\begin{array}{l}\text { Leptomeningeal } \\
\text { change in brain MRI }\end{array}$ & $\begin{array}{l}\text { Linear } \\
\text { enhancement }\end{array}$ & Negative & Linear enhancement & Negative & $\begin{array}{l}\text { Small patched } \\
\text { enhancement }\end{array}$ \\
\hline CSF cell examination & $\begin{array}{l}\text { Adenocarcinoma } \\
\text { cell }\end{array}$ & Adenocarcinoma cell & Adenocarcinoma cell & $\begin{array}{l}\text { Adenocarcinoma } \\
\text { cell }\end{array}$ & $\begin{array}{l}\text { Adenocarcinoma } \\
\text { cell }\end{array}$ \\
\hline T790M mutation & Negative & Negative & Negative & Negative & Negative \\
\hline $\begin{array}{l}\text { Time to onset for } \\
\text { osimertinib orally } \\
\text { (days) }\end{array}$ & 7 & 10 & 7 & 5 & 8 \\
\hline
\end{tabular}

temozolomide treatment, but the disease was not controlled after cranial radiotherapy. T790M gene testing was still negative at that time. Then the patient developed nausea, vomiting, and blurred vision, and the therapy was replaced by osimertinib orally $80 \mathrm{mg}$ once a day. Her symptoms reduced gradually after 1 week.

The remaining 3 patients did not have LM at the first diagnosis. One patient had 19 exon deletion and T790M negative. His first line of treatment was chemotherapy combined with erlotinib, and he was given 64 months of progression-free survival for the first time. After the diagnosis of leptomeningeal progression, the patients have replaced it with osimertinib orally $80 \mathrm{mg}$ once a day, and the psychiatric symptoms were significantly relieved after 1 week.
According to the results of genetic testing, LM occurred in the other two patients 3 months after gefitinib and 4 months after icotinib. Osimertinib was used as orally $80 \mathrm{mg}$ once a day when the neurological symptoms were not relieved after applicating analgesia, cranial pressure reduction, and systemic chemotherapy method. In the first case, dizziness, headache, nausea, and vomiting symptoms disappeared, and blurred vision was relieved 5 days after osimertinib treatment, neurological symptoms were relieved in the other patient 8 days after osimertinib treatment (Table 1).

All five patients were followed up and survived continuously in June 2019. The remission time was 10, 7, 7, 5, 4 months respectively, and the total survival time was 74 , 7, 27, 18, and 4 months correspondingly (Table 1). The side 

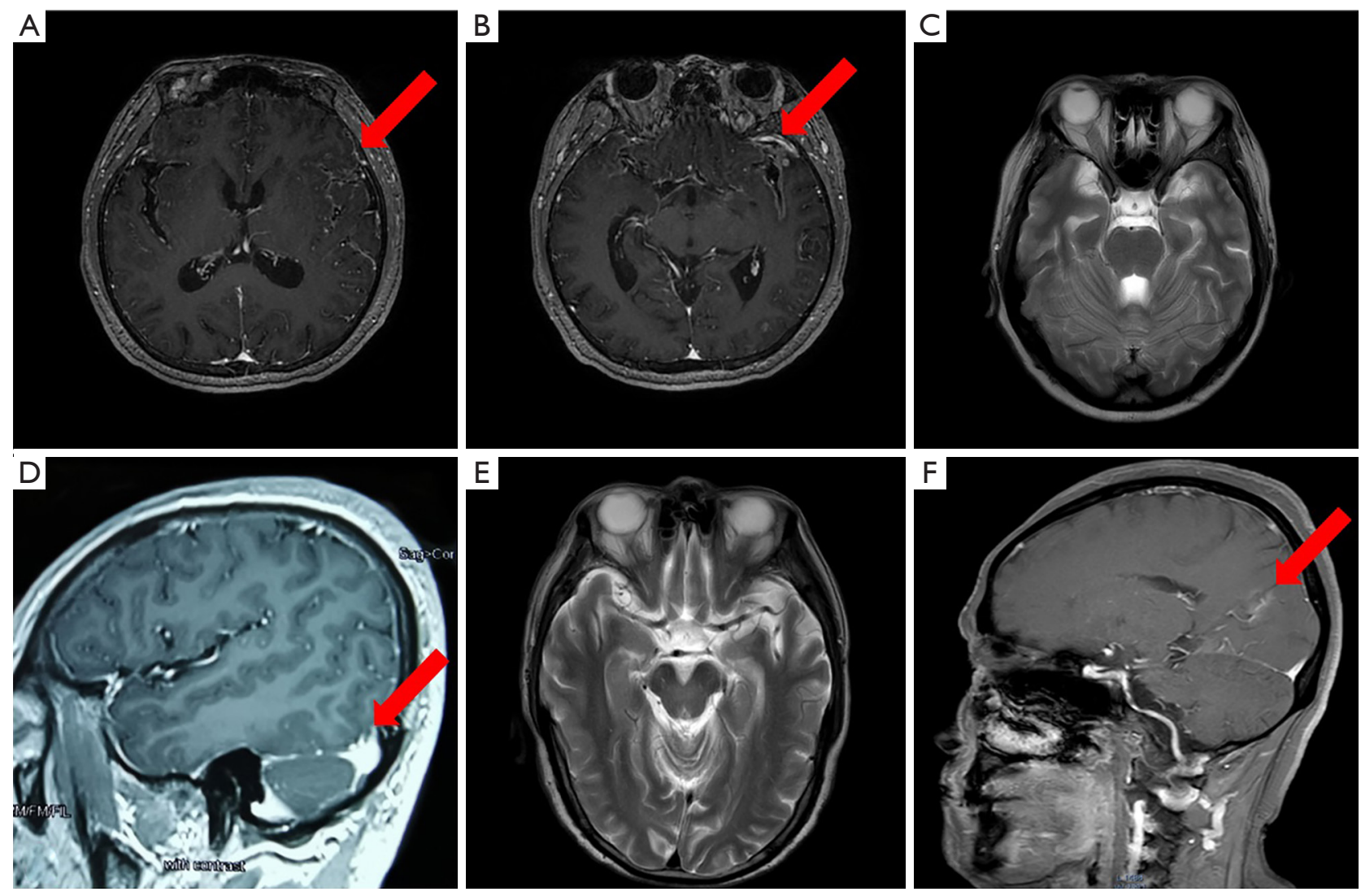

Figure 1 Brain MRI: abnormal line-like enhancement can be seen in the leptomeningeal of both temporal lobes (A,B). None abnormal enhancement lines in leptomeningeal and brain parenchyma, none hypodense edema area (C). Multiple enhancement lines in both cerebellar sulci (D). Cerebral ischemia in periventricular area, none abnormal enhancement lines in leptomeningeal and brain parenchyma (E). Small patched enhancement in occipital lobe $(\mathrm{F})$.

Table 2 CEA, glucose, protein in CSF

\begin{tabular}{llcc}
\hline Patient & CEA $(\mathrm{ng} / \mathrm{mL})$ & Glucose $(\mathrm{mmol} / \mathrm{L})$ & Protein $(\mathrm{g} / \mathrm{L})$ \\
\hline 1 & 352.65 & 1.21 & 0.57 \\
2 & 369.54 & 2.0 & 0.48 \\
3 & 255.75 & 2.12 & 0.89 \\
4 & 285.7 & 1.30 & 0.65 \\
5 & 265.25 & 1.05 & 0.7 \\
\hline
\end{tabular}

Normal range of CEA: $0-5 \mathrm{ng} / \mathrm{mL}$; normal range of glucose: 2.20-3.90 mmol/L; normal range of protein: $0.15-0.45 \mathrm{~g} / \mathrm{L}$.

effects were not increased.

\section{Discussion}

The incidence of LM in NSCLC was $20-40 \%$ (7), especially high to $84-96 \%$ in adenocarcinoma subtype because of the proportion was high in recent years (8-10). LM was also an important indicator for poor prognosis. Once $\mathrm{LM}$ occurred, the median survival time of the patient was only 4-8 months (11). The diagnosis of LM of lung cancer majorly depended on clinical manifestations, including increased intracranial pressure and leptomeningeal stimulating symptoms. It was also accompanied by cranial nerve and spinal nerve root involvement (12). The imaging examination was often negative (8), especially in patients with diffusely LM (13). Some authors had reported that enhanced cranial MRI could display linear and nodular enhancement in meninges (3). In our study, 5 patients presented dizziness and headache due to increased intracranial pressure, and 3 patients presented blurred vision and decreased vision, namely, cranial nerve involvement. Multiple brain MRI examinations showed no abnormality in 2 patients 
and revealed leptomeningeal enhancement with $\mathrm{LM}$ in 3 patients, which were consistent with the literature reports. Diagnosis of LM needs CSF examination. However, the sensitivity and effectiveness of CSF cytology were only $75 \%$ and $83.3 \%$ (14). The biochemistry results of CSF had some diagnostic value for LM. It had been reported that the levels of white blood cells and protein in CSF increased and the levels of glucose decreased in patients with LM of NSCLC $(15,16)$. Carcinoembryonic antigen (CEA) in CSF could also be used as a reference for the diagnosis of LM. The level of CEA in CSF of patients with LM of primary lung cancer was significantly higher than that of other tumor species. Combined with the changes in CEA level in patients' blood and CSF, the positive rate of detecting LM could be improved (17). In our study, CEA in blood and CSF increased significantly in 5 patients, and LM could be diagnosed by combining clinical manifestations and imaging examination, which was consistent with literature reports.

The treatment of LM was different from that of brain parenchymal metastasis in lung cancer. There had no unified standard treatment method yet. Systemic chemotherapy, targeted therapy, intrathecal or ventricular chemotherapy, and radiotherapy were common methods to be used in the clinical treatment. Although first-generation EGFR-TKI targeted drugs are now widely used as first-line treatment for patients with advanced NSCLC with EGFR mutation, their efficacy for meninges metastasis was still limited due to their weak blood-brain barrier permeability (18). More than $30 \%$ of patients with intracranial metastasis had been reported to have disease progression during or after TKI treatment (19). Some authors reported that the first-generation EGFR-TKI drugs, such as gefitinib and erlotinib, could improve the efficacy by highdose pulsed administration, but the effect was still not significant, and the median survival time of patients was not significantly prolonged (20-23). Comparing with the platinum-containing two-drug combined regimen, the third generation of EGFR-TKI drugs such as osimertinib showed significantly increased the median PFS (11.7 vs. 5.6 months, $\mathrm{P}=0.004)$ for the T790M mutation-positive NSCLC patients with brain metastasis in the results of AURA3 phase III clinical trial (24). Among the patients in advanced NSCLC who were treated for the first time, whether the T790M mutation was negative or positive, the efficacy of osimertinib was better than that of first-generation TKI drugs (8). In preclinical studies, osimertinib was revealed significant efficacy in mouse models of brain metastasis (25), and it had better blood-brain barrier permeability than gefitinib and afatinib (26).

The BLOOM phase I clinical study had assessed the reactivity and safety of osimertinib and compared its advantage to the earlier TKI drug treatment in patients with LM (27). Preliminary results showed that 23 out of 32 patients benefited, 10 had imaging changes, and 13 had stable lesions after 12 weeks of treatment in 32 patients. In this study, 11 patients tested positive for T790M mutation, and 21 patients failed the T790M mutation test. Patients who fail the genetic test included T790M negative; this means that osimertinib may also be effective in T790M negative patients. Chalmers et al. (28) reported that patients with $T 790 M$ negative remained stable for 1 year when treated with osimertinib in advanced NSCLC. Facchinetti et al. (29) also reported that osimertinib was effective in the treatment of meningeal metastasis of T790M negative NSCLC patients by imaging changes in brain lesions. According to diagnosis and treatment consensus of brain and LM from lung cancer in China (30), osimertinib also can be used for brain (leptomeningeal) metastases as the disease progressing after TKI treatment without the T790M mutation. In our study, EGFR mutation was positive, and T790M mutation was negative by NGS in puncture tissues and peripheral blood in all the 5 patients when diagnosed as LM. After treatment by osimertinib, neurological symptoms were significantly relieved, clinical benefits were significantly obtained, and survival period was prolonged. Due to the presence of the blood-brain barrier, the acquisition of gene detection for intracranial lesions could be affected, and even false-negative results were obtained. Huang et al. (31) reported that in patients with $\mathrm{LM}$, there is a difference between CSF and blood gene detection, and the T790M of blood gene detection may be negative, while that of CSF is contrary. Our five patients had not repeated to detect EGFR mutation in CSF, and it was questionable whether there was a false negative to T790M. Peripheral blood gene detection is used in clinical to overcome the heterogeneity of tumor tissues in recent years. Therefore, the false-negative rate of T790M mutation detection can be reduced by simultaneous detection of EGFR gene mutation and T790M gene mutation in CSF in patients suspected of LM.

\section{Conclusions}

In conclusion, it is difficult for patients with LM of NSCLC to be diagnosed at an early stage, and comprehensive 
consideration should be given to the clinical manifestations, CSF cytology, laboratory examination, and imaging examination. Our study shows that even if T790M mutation is negative or cannot be accurately measured, early applicating of osimertinib shall be considered, and it may obtain better efficacy and clinical benefits for patients with LM of lung cancer, especially for patients with LM of NSCLC with EGFR gene mutation.

\section{Acknowledgments}

Funding: The project is supported by Health Bureau of Anhui Province, China (No. 09B114).

\section{Footnote}

Conflicts of Interest: The authors have no conflicts of interest to declare.

Etbical Statement: The authors are accountable for all aspects of the work in ensuring that questions related to the accuracy or integrity of any part of the work are appropriately investigated and resolved. Patients were prospectively reviewed on our retrospective analysis and followed for 1 year. This retrospective analysis was approved by The Clinical Medical Research Ethics Committee of The First Affiliated Hospital of Anhui Medical University (No. Quick PJ 2019-13-11). The privacy consent was achieved by all patients.

\section{References}

1. Kim H, Lee EM. A Retrospective analysis of the clinical outcomes of leptomeningeal metastasis in patients with solid tumors. Brain Tumor Res Treat 2018;6:54-9.

2. Li YS, Jiang BY, Yang JJ, et al. Leptomeningeal metastases in patients with NSCLC with EGFR mutations. J Thorac Oncol 2016;11:1962-9.

3. Lee SJ, Lee JI, Nam DH, et al. Leptomeningeal carcinomatosis in non-small cell lung cancer patients: Impact on survival and correlated prognostic factors. J Thorac Oncol 2013;8:185-91.

4. Kuiper JL, Hendriks LE, van der Wekken AJ, et al. Treatment and survival of patients with EGFR-mutated non-small cell lung cancer and leptomeningeal metastasis: a retrospective cohort analysis. Lung Cancer 2015;89:255-61.

5. Mok TS, Wu YL, Ahn MJ, et al. Osimertinib or platinumpemetrexed in EGFR T790M- positive lung cancer. N
Engl J Med 2017;376:629-40.

6. Soria JC, Ohe Y, Vansteenkiste J, et al. Osimertinib in untreated EGFR-mutated advanced non-small cell lung cancer. N Engl J Med 2018;378:113-25.

7. Yuan Y, Tan C, Li M, et al. Activity of pemetrexed and high-dose gefitinib in an EGFR mutated lung adenocarcinoma with brain and leptomeningeal metastasis after response to gefitinib. World J Surg Oncol 2012;10:235.

8. Park JH, Kim YJ, Lee JO, et al. Clinical outcomes of leptomeningeal metastasis in patients with non-small cell lung cancer in the modern chemotherapy era. Lung Cancer 2012;76:387-92.

9. Gwak HS, Joo J, Kim S, et al. Analysis of treatment outcomes of intraventricular chemotherapy in 105 patients for leptomeningeal carcinomatosis from non-small cell lung cancer. J Thorac Oncol 2013;8:599-605.

10. Morris PG, Reiner AS, Szenberg OR, et al. Leptomeningeal metastasis from non-small cell lung cancer: Survival and the impact of whole brain radiotherapy. J Thorac Oncol 2012;7:382-5.

11. Ma C, Jiang R, Li JZ, et al. Research progress of lung cancer with leptomeningeal metastasis. Zhongguo Fei Ai Za Zhi 2014;17:695-700.

12. Le Rhun E, Tu Q, De Carvalho Bittencourt M, et al. Detection and quantification of CSF malignant cells by the CellSearch technology in patients with melanoma leptomeningeal metastasis. Med Oncol 2013; 30:538.

13. Remon J, Le Rhun E, Besse B. Leptomeningeal carcinomatosis in non-small cell lung cancer patients: A continuing challenge in the personalized treatment era. Cancer Treat Rev 2017;53:128-37.

14. $\mathrm{Lv} \mathrm{Y}, \mathrm{Mu} \mathrm{N}, \mathrm{MaC}$, et al. Detection value of tumor cells in cerebrospinal fluid in the diagnosis of meningeal metastasis from lung cancer by immuno-FISH technology. Oncol Lett 2016;12:5080-4.

15. Hyun JW, Jeong IH, Joung A, et al. Leptomeningeal metastasis: Clinical experience of 519 cases. Eur J Cancer 2016;56:107-14.

16. Sahebjam S, Forsyth PA, Smalley KS, et al. Experimental Treatments for Leptomeningeal Metastases From Solid Malignancies. Cancer Control 2017;24:42-6.

17. Grunnet M, Sorensen JB. Carcinoembryonic antigen (CEA) as tumor marker in lung cancer. Lung Cancer 2012;76:138-43.

18. Flippot R, Biondani P, Auclin E, et al. Activity of EGFR tyrosine kinase Inhibitors in NSCLC with refractory leptomeningeal metastases. J Thorac Oncol 
2019;14:1400-7.

19. Heon S, Yeap BY, Britt GJ, et al. Development of central nervous system metastases in patients with advanced non-small cell lung cancer and somatic EGFR mutations treated with gefitinib or erlotinib. Clin Cancer Res 2010;16:5873-82.

20. Grommes C, Oxnard GR, Kris MG, et al. "Pulsatile" high-dose weekly erlotinib for CNS metastases from EGFR mutant non-small cell lung cancer. Neuro Oncol 2011;13:1364-9.

21. Togashi Y, Masago K, Fukudo M, et al. Efficacy of increased-dose erlotinib for central nervous system metastases in non-small cell lung cancer patients with epidermal growth factor receptor mutation. Cancer Chemother Pharmacol 2011;68:1089-92.

22. Milton DT, Azzoli GC, Heelan RT, et al. A phase I/ II study of weekly high-dose erlotinib in previously treated patients with nonsmall cell lung cancer. Cancer 2006; 107:1034-41.

23. Kawamura T, Hata A, Takeshita J, et al. High-dose erlotinib for refractory leptomeningeal metastases after failure of standard-dose EGFR-TKIs. Cancer Chemother Pharmacol 2015;75:1261-6.

24. Wu YL, Ahn MJ, Garassino MC, et al. CNS efficacy of Osimertinib in patients with T790M-positive advanced non-small- cell lung cancer: Data from a randomized phase III trial (AURA3). J Clin Oncol 2018;36:2702-9.

25. Nanjo S, Ebi H, Arai S, et al. High efficacy of third

Cite this article as: $\mathrm{Hu} \mathrm{X}$, Chen $\mathrm{W}$, Li X, Zhao C, Zhang C, Xiong F, Wu H. Clinical efficacy analysis of Osimertinib treatment for a patient with leptomeningeal metastasis of $E G F R+$ non-small cell lung cancer without the T790M mutation. Ann Palliat Med 2019;8(5):525-531. doi: 10.21037/ apm.2019.10.13 generation EGFR inhibitor AZD9291 in a leptomeningeal carcinomatosis model with EGFR-mutant lung cancer cells. Oncotarget 2016;7:3847-56.

26. Ballard P, Yates JW, Yang Z, et al. Preclinical comparison of osimertinib with other EGFR-TKIs in EGFR-mutant NSCLC brain metastases models, and early evidence of clinical brain metastases activity. Clin Cancer Res 2016;22:5130-40.

27. Yang JC, Cho BC, Kim D, et al. Osimertinib for patients with leptomeningeal metastases from EGFR-mutant nonsmall cell lung cancer: updated results from the BLOOM study. J Clin Oncol 2017;35:abstr 2020.

28. Chalmers A, Jensen L, Akerley W. Durable response to osimertinib in EGFR mutated T790M wildtype non-small cell lung cancer with leptomeningeal metastases: A case report. Lung Cancer 2017;114:68-9.

29. Facchinetti F, Bozzetti F, Minari R, et al. Meeting with triumph and disaster: Osimertinib in T790M-unknown CNS progression in EGFR-mutated non-small cell lung cancer. Tumori 2018;104:NP29-33.

30. Wu YL, Lu S, Wang CL, et al. Diagnosis and Treatment Consensus of Brain and Leptomeningeal Metastasis from Lung Cancer. Xun Zheng Yi Xue 2018;18:193-200.

31. Huang R, Ge M, Zhou X, et al. Epidermal Growth Factor Receptor Mutation Detection in Cerebrospinal Fluid of Lung Adenocarcinoma Patients with Leptomeningeal Metastasis. Cancer Biother Radiopharm 2019;34:128-33. 